\title{
On Robotic Optimal Path Planning in Polygonal Regions With Pseudo-Euclidean Metrics
}

\author{
Zheng Sun, Member, IEEE, and John H. Reif, Fellow, IEEE
}

\begin{abstract}
This paper presents several results on some costminimizing path problems in polygonal regions. For these types of problems, an approach often used to compute approximate optimal paths is to apply a discrete search algorithm to a graph $\mathcal{G}_{\epsilon}$ constructed from a discretization of the problem; this graph is guaranteed to contain an $\epsilon$-good approximate optimal path, i.e., a path with a cost within $(1+\epsilon)$ factor of that of an optimal path, between given source and destination points. Here, $\epsilon>0$ is the user-defined error tolerance ratio. We introduce a class of piecewise pseudo-Euclidean optimal path problems that includes several non-Euclidean optimal path problems previously studied and show that the BUSHWHACK algorithm, which was formerly designed for the weighted region optimal path problem, can be generalized to solve any optimal path problem of this class. We also introduce an empirical method called the adaptive discretization method that improves the performance of the approximation algorithms by placing discretization points densely only in areas that may contain optimal paths. It proceeds in multiple iterations, and in each iteration, it varies the approximation parameters and fine tunes the discretization.
\end{abstract}

Index Terms-Computational geometry, mobile robots, motion planning, optimization.

\section{INTRODUCTION}

\section{A. Motivations and Previous Works}

In applications such as robotic motion planning and geographical information systems, there arise geometric optimal path problems where one has to find a minimal cost path connecting two given points $s$ and $t$ (i.e., the single-source single-destination problem). In the past two decades, these problems have been extensively studied (see [3] for a review). One common assumption made by many previous studies on these problems is that, for any $s$ and $t$, an optimal path from $s$ to $t$ is a straight-line segment $\overline{s t}$ if $\overline{s t}$ lies entirely inside the free space, i.e., the portion of the robot's workspace not occupied by obstacles.

Manuscript received May 3, 2006. This work was supported in part by the National Science Foundation (NSF) under Grants CCF-0432038 and CCF0523555, and in part by RGC under Grant HKBU2107/04E. This paper was presented in part at the 12th Annual International Symposium on Algorithms and Computation [1] and at the 14th International Symposium on Fundamentals of Computation Theory [2]. This paper was recommended by Associate Editor S. E. Shimony.

Z. Sun is with Google Inc., Mountain View, CA 94043 USA (e-mail: sunzheng@gmail.com).

J. H. Reif is with the Department of Computer Science, Duke University, Durham, NC 27708-0129 USA (e-mail: reif@cs.duke.edu).

Color versions of one or more of the figures in this paper are available online at http://ieeexplore.ieee.org.

Digital Object Identifier 10.1109/TSMCB.2007.896021
In recent years, there has been increasing attention and motivation on path planning problems with various non-Euclidean cost metrics on paths. If the free space consists of multiple regions with different cost metrics, the straight-line segment $\overline{s t}$ may no longer be an optimal path from $s$ to $t$ even if $\overline{s t}$ lies in free space. Therefore, many techniques developed in previous path planning works are no longer valid.

In the weighted region optimal path problem [4]-[10], each region of a polygonal subdivision of 2-D space is assigned with a distinct unit weight. Such a space can be used to model an area consisting of different geographical features such as deserts, forests, grasslands, and lakes, in which the maximum traveling speed of the robot is different. The goal is to find for two given points $s$ and $t$ an optimal path from $s$ to $t$ with the minimum weighted length. Two other examples of nonEuclidean optimal path problems are the flow path problem, which computes a time-minimizing path for a point robot with a fixed maximum velocity in $n$ triangular regions with uniform flows (see [11]), and the anisotropic optimal path problem, which computes an energy-minimizing path for a mobile robot on a terrain consisting of $n$ terrain faces each defined with ranges of forbidden traversal directions due to power limitations or overturn danger (see [12]-[16]).

Compared with the 2-D Euclidean shortest path problem, which can be solved in $O(n \ln n)$ time (e.g., see [17]), these optimal path problems are believed to be very difficult. Although no computational complexity result has been established for computing exact solutions for these problems, it has been proved that the combinatorial complexity of optimal paths for each of these problems could be much higher than that of Euclidean shortest paths (see [4], [11], and [16]). Therefore, much of the effort has been focused on $\epsilon$-approximation algorithms that can guarantee to find $\epsilon$-good approximate optimal paths. For any two points $s$ and $t$ in space, we call a path $p$ connecting $s$ and $t$ an $\epsilon$-good approximate optimal path if $\|p\|<$ $(1+\epsilon) \cdot\left\|p_{\text {opt }}(s, t)\right\|$, where $p_{\text {opt }}(s, t)$ denotes an optimal path from $s$ to $t$, and $\|\cdot\|$ represents the cost of a path (according to the defined cost metric). Equivalently, we say that $p$ is $p_{\text {opt }}(s, t)$ 's $\epsilon$-approximation.

To solve the weighted region optimal path problem, a number of previous works [5]-[7], [10] used a (uniform or logarithmic) discretization of the problem based on edge subdivision, and Dijkstra's algorithm to find a shortest path between $s$ and $t$ in the weighted graph $\mathcal{G}$ induced by discretization. This shortest path, which we call an optimal discrete path, is then converted to a path in the original continuous space as an approximation of a "true" optimal path. 
Aleksandrov et al. [8] proposed a logarithmic discretization scheme that generates a discretization bound containing an $\epsilon$-good approximate optimal path between $s$ and $t$ for any user-defined error tolerance $\epsilon>0$. Their algorithm places $m=O((1 / \epsilon) \ln (1 / \epsilon))$ representative points, which are called Steiner points, on each boundary edge and then applies a "pruned" version of Dijkstra's algorithm to compute in $O((n / \epsilon)((1 / \sqrt{\epsilon})+\ln n) \ln (1 / \epsilon))$ time an optimal discrete path in the resulting graph $\mathcal{G}_{\epsilon}$. Sun and Reif [9] introduced an alternative discrete search algorithm called BUSHWHACK that can be applied to any edge-subdivision-based discretization of the weighted region optimal path problem. BUSHWHACK can compute an optimal discrete path more efficiently by avoiding accessing most of the edges in $\mathcal{G}_{\epsilon}$. Combined with the discretization scheme in [8], it can compute an $\epsilon$-good approximate optimal path in $O((n / \epsilon)(\ln (1 / \epsilon)+\ln n) \ln (1 / \epsilon))$ time.

Aleksandrov et al. [10] later improved the above time complexity by another factor of $O(1 / \sqrt{\epsilon})$. They achieved this by using a novel discretization method that places Steiner points on bisectors of triangular regions instead of on boundary edges. This discretization method is able to reduce the number of Steiner points by a factor of $O(1 / \sqrt{\epsilon})$ while still guaranteeing the same $\epsilon$ error bound. They then applied a discrete algorithm similar to BUSHWHACK to compute an optimal discrete path in such a discretization.

Approximation algorithms using edge-subdivision-based discretizations have also been developed for two extensions of the weighted region optimal path problem: the flow path problem [11] and the anisotropic optimal path problem [15], [16]. And a general and improved discretization method for this type of problems has been discussed [18].

In the remainder of this paper, we will mainly discuss techniques for approximation algorithms using this discretization approach. Since an optimal discrete path from $s$ to $t$ in $\mathcal{G}_{\epsilon}$ is used as an approximation for a true optimal path, the phrases "optimal discrete path" and "approximate optimal path" are used interchangeably and are both denoted by $p_{\text {opt }}^{\prime}(s, t)$. Please refer to [10] for a detailed discussion on how to construct $\mathcal{G}_{\epsilon}$ to guarantee an $\epsilon$-good approximate optimal path.

In this paper, we assume that none of the regions is "impassable," because for approximation algorithms adopting this discretization approach, impassable regions can be easily handled (by not connecting the Steiner points on the boundary of the same impassable region). This is also the case for the flow path and the anisotropic optimal path problem, where the cost metric is direction dependent and so is the notion of passability.

\section{B. Our Contributions}

In this paper, we present the following results on finding $\epsilon$-good approximate optimal paths using edge-subdivisionbased discretizations. These results can be applied to various discretization schemes for a wide range of non-Euclidean optimal path problems.

1) Generalized BUSHWHACK Algorithm: Just like Dijkstra's algorithm, BUSHWHACK [9] is used to compute optimal discrete paths in a graph $\mathcal{G}_{\epsilon}$ generated by a discretization scheme. However, Dijkstra's algorithm applies to any arbitrary weighted graph, while BUSHWHACK is adept at finding optimal discrete paths in graphs derived from 2-D spaces consisting of weighted regions. In particular, BUSHWHACK exploits the fact that two optimal discrete paths originating from $s$ cannot intersect in the interior of a region, and therefore, two intersecting graph edges in $\mathcal{G}_{\epsilon}$ cannot be both useful. Here, we say that a graph edge is useful if it is part of an optimal discrete path originating from $s$.

However, unlike the pruned Dijkstra's algorithm proposed by Aleksandrov et al. [7], [10] and other $\epsilon$-approximation algorithms [4], [5], BUSHWHACK does not rely too much on the geometric property that an optimal path would obey "Snell's law" when it crosses region boundaries, which is a property only applicable to the weighted region optimal path problem. ${ }^{1}$ Therefore, BUSHWHACK can be generalized (after modifications) to computing approximate optimal paths in 2-D spaces with a wide range of possible geometric properties. In Section II, we characterize a class of piecewise pseudoEuclidean spaces for which the generalized BUSHWHACK algorithm can be applied to compute optimal paths. The main restriction on the cost metrics of spaces in this class is that, for any point $v$ on the boundary of a region, any boundary edge opposing this point can be divided into a small number (bounded by a constant) of segments such that in each segment the distance from/to $v$ (as defined by the cost metric) is monotonic.

2) Adaptive Discretization Method: A traditional approximation algorithm proceeds in a one-step manner; it constructs from the original space a graph $\mathcal{G}_{\epsilon}$ and then computes (with a discrete search algorithm such as Dijkstra's algorithm) an optimal discrete path in $\mathcal{G}_{\epsilon}$. We call this method the fixed discretization method. For a single-source single-destination problem, this method is rather inefficient in that, although the goal is to find an $\epsilon$-good approximate optimal path $p_{\text {opt }}^{\prime}(s, t)$ from $s$ to $t$, it actually computes an $\epsilon$-good approximate optimal path from $s$ to any point $v$ in $\mathcal{G}_{\epsilon}$ as long as the cost of such a path is less than $\left\|p_{\text {opt }}^{\prime}(s, t)\right\|$. Much of the effort is unnecessary as most of these points would not help in finding an $\epsilon$-good approximate optimal path from $s$ to $t$.

We use flight ticket booking as an example. When trying to find the cheapest flight from Hong Kong to San Francisco with one stop (supposing no direct flight is available), a travel agent does not need to consider Mexico City as a candidate for the connecting airport if she knows the following: 1) by no means the cheapest flight from Hong Kong to San Francisco with one stop would cost as much as $\$ 1300 ; 2$ ) any direct flight from Hong Kong to Mexico City costs no less than $\$ 1000$; and 3) any direct flight from Mexico City to San Francisco costs no less than $\$ 500$. Therefore, she does not need to find out the exact prices of the direct flights from Hong Kong to Mexico City and from Mexico City to San Francisco, which saves two queries to the ticketing database.

\footnotetext{
${ }^{1}$ Although for other cost metrics we could define similar local optimality conditions just like the Snell's Law for the weighted region optimal path problem, the equations for computing the "bending points" may not be easily solvable. This is the case for the flow path problem and the anisotropic optimal path problem.
} 
Analogously, we do not need to compute $p_{\text {opt }}^{\prime}(s, v)$ and $p_{\text {opt }}^{\prime}(v, t)$ for a point $v \in \mathcal{G}_{\epsilon}$ if we know in advance that $v$ does not connect any optimal discrete path between $s$ and $t$. However, while the travel agent can rely on knowledge she previously gained, the approximation algorithms using the fixed discretization method have no prior knowledge to draw upon. In Section III, we discuss a multiround discretization method that we call the adaptive discretization method. It starts with a coarse discretization $\mathcal{G}^{\prime}=\mathcal{G}_{\epsilon_{1}}$ for some $\epsilon_{1}>\epsilon$ and adaptively refines $\mathcal{G}^{\prime}$ until it is guaranteed to contain an $\epsilon$-good approximate optimal path from $s$ to $t$. Approximate optimal path information acquired in each round is used to identify the "useless" areas where no optimal path from $s$ to $t$ will pass through and therefore no further refinement (i.e., inserting more Steiner points) is needed in the next round.

Intuitively, this method is similar to the well-known $\mathrm{A}^{*}$ algorithm but with considerable distinctions. In particular, this method does not rely on a good heuristic evaluation on path costs to achieve performance improvement (over the standard Dijkstra's algorithm); instead, it acquires one with bounded error via prior iterations.

\section{Generalized BUSHWHACK AlgORITHM}

\section{A. Review of Bushwhack}

In this subsection, we give a brief review of the BUSHWHACK algorithm [9], which was designed for the weighted region optimal path problem. We refer readers to [9] for a more detailed description. In the subsequent subsections, we will show how to generalize this algorithm for a wide class of optimal path problems.

Given an instance of the weighted region optimal path problem, BUSHWHACK starts with constructing from the original continuous space a weighted graph $\mathcal{G}_{\epsilon}\left(V^{\prime}, E^{\prime}\right)$ by placing Steiner points on boundary edges. We call both the Steiner points and the vertices of the regions discrete points. The node set $V^{\prime}$ of $\mathcal{G}_{\epsilon}$ contains all discrete points, and the edge set $E^{\prime}$ of $\mathcal{G}_{\epsilon}$ contains every graph edge $\left(v_{1}, v_{2}\right)$ such that $v_{1}$ and $v_{2}$ are on the border of the same region. For any graph edge $\left(v_{1}, v_{2}\right)$ (corresponding to segment $\overline{v_{1} v_{2}}$ in the original space), the weight $w\left(v_{1}, v_{2}\right)$ assigned is the weighted length of segment $\overline{v_{1} v_{2}}$ in the original weighted space.

Our BUSHWHACK algorithm exploits not only the graph theoretic properties of each edge $\left(v_{1}, v_{2}\right)$ but also the geometric properties of the straight-line segment $\overline{v_{1} v_{2}}$ (e.g., whether this segment intersects with another segment and whether it lies on a boundary edge or across a region). Therefore, in the following discussion, we will use $\overline{v_{1} v_{2}}$ to denote the graph edge connecting $v_{1}$ and $v_{2}$ as well as the corresponding straight-line segment. When a discrete path is converted to the corresponding path in the original continuous space, each edge $\overline{v_{1} v_{2}}$ is converted to a subpath. It is important to note however that although for the weighted region optimal path problem such a subpath is a straight-line path, it may not be the case for other problems that we shall study.

Dijkstra's algorithm can be used to compute an optimal discrete path in $\mathcal{G}_{\epsilon}$. However, it does not take advantage of the following property when searching for optimal discrete paths.

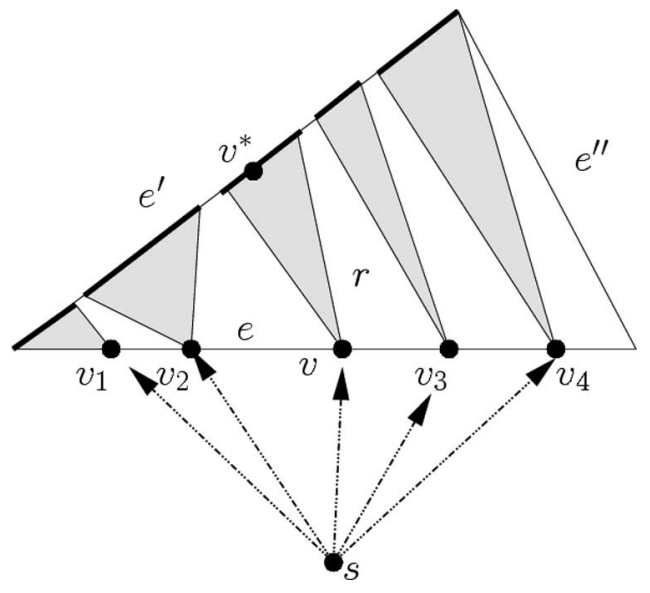

Fig. 1. Point $s$ is the source point, and $v, v_{1}, \ldots, v_{4}$ are the discovered points on edge $e$. Edge $e^{\prime}$ is divided into intervals (marked by solid segments), one for each discovered point on $e$.

1) Property 1: Two optimal discrete paths that originate from the same source point cannot intersect in the interior (i.e., excluding the boundary) of any region.

One implication of Property 1 is that, if two edges $\overline{v_{1} v_{2}}$ and $\overline{u_{1} u_{2}}$ of $\mathcal{G}_{\epsilon}$ intersect inside region $r$, they cannot be both useful. To exploit this property, BUSHWHACK maintains a list $\operatorname{ILIST}_{e, e^{\prime}}$ of intervals for each pair of boundary edges $e$ and $e^{\prime}$ that are on the border of the same region $r$. We use PLIST $_{e}$ to denote the list of all discovered points on $e$, where a discrete point $v$ is said to be discovered if an optimal discrete path $p_{\text {opt }}^{\prime}(s, v)$ has been determined. ILIST $T_{e, e^{\prime}}$ contains for each discovered point $v \in \operatorname{PLIST}_{e}$ an interval $I_{v, e, e^{\prime}}$ defined as in the following.

Definition 1: Let $r$ be a region of $S$, and let $e$ and $e^{\prime}$ be two boundary edges of $r$. For any discovered point $v \in e$ that is not incident to $e^{\prime}$, the interval $I_{v, e, e^{\prime}}$ (see Fig. 1) is defined to be

$$
\begin{aligned}
I_{v, e, e^{\prime}}=\left\{v^{*} \in e^{\prime} \mid d_{r}\left(v, v^{*}\right)+\left\|p_{\mathrm{opt}}^{\prime}(s, v)\right\| \leq d_{r}\left(v^{\prime}, v^{*}\right)\right. \\
\left.+\left\|p_{\mathrm{opt}}^{\prime}\left(s, v^{\prime}\right)\right\| \quad \forall v^{\prime} \in \mathrm{PLIST}_{e}\right\} .
\end{aligned}
$$

Here, $d_{r}\left(v, v^{*}\right)$ is the region distance between $v$ and $v^{*}$, which is defined to be the weighted length of segment $\overline{v v^{*}}$. (We will give a more general definition for region distance in the next subsection.)

Intuitively, for any discrete point $v^{*}$ on $e^{\prime}, v^{*} \in I_{v, e, e^{\prime}}$ if and only if path $p_{\text {opt }}^{\prime}(s, v)+\overline{v v^{*}}$ is the least costly path among all paths from $s$ to $v^{*}$ that are one-segment extensions of optimal discrete paths originating from $s$ to discovered points on $e$. Intervals in $\operatorname{ILIST}_{e, e^{\prime}}$ partition the discrete points on boundary edge $e^{\prime}$ into contiguous segments, as shown in Fig. 1. If necessary, an interval $I_{v, e, e^{\prime}}$ is further divided into two monotonic intervals by the perpendicular point $v_{h}$ of $v$ on $e^{\prime}$ if $v_{h}$ is located inside $I_{v, e, e^{\prime}}$. The goal is to make the distances from $v$ to all discrete points in $I_{v, e, e^{\prime}}$ monotonically increasing or decreasing. (We will explain later why this is necessary.)

We say that edge $\overline{v v^{*}}$ is associated with interval list $\operatorname{ILIST}_{e, e^{\prime}}$ if $v \in e$ and $v^{*} \in I_{v, e, e^{\prime}}$. It is easy to see that any edge $\frac{v, e^{*}}{v v^{*}}$ crossing region $r$ is useful only if it is associated with an interval list inside $r$. If $m$ is the number of Steiner points placed on each boundary edge, the total number of edges associated with 
interval lists inside a region $r$ is $\Theta(m)$. Dijkstra's algorithm, on the other hand, has to consider all $\Theta\left(m^{2}\right)$ edges inside $r$. By avoiding accessing most of the useless edges, BUSHWHACK takes only $O(n m \ln n m)$ time to compute an optimal discrete path from $s$ to $t$ as compared to $O\left(n m^{2}+n m \ln n m\right)$ time for Dijkstra's algorithm.

Note that Mitchell and Papadimitriou [4] also utilized Property 1 by defining intervals of optimality. For a discussion on the difference between intervals of optimality (which are continuous) and our "discrete" intervals, please see [9].

There are two main parts of the BUSHWHACK algorithm. The first is to maintain the interval list $\operatorname{ILIST}_{e, e^{\prime}}$ for each pair of boundary edges $e$ and $e^{\prime}$ that are on the border of the same region. The intervals in $\operatorname{ILIST}_{e, e^{\prime}}$ are dynamic data structures; they may get modified every time a discrete point $v \in e$ is discovered. The second part is to insert candidate optimal paths into a path list QLIST, which is a priority list similar to the one used by Dijkstra's algorithm. Each candidate optimal path extends by one segment an optimal discrete path from $s$ to some discovered point and will be tested for optimality (and subsequently extracted from QLIST) once it becomes the minimum cost path in QLIST.

\section{B. Definitions}

Let $r$ be a closed triangular region associated with a cost metric defined on paths. For any two points $x$ and $y$ in $r$, a facewise optimal path, which is denoted by $p_{\mathrm{opt}}^{r}(x, y)$, is a costminimizing path (under the defined path cost metric) among all paths connecting $x$ and $y$ and lying completely inside $r$. We define the region distance $d_{r}(x, y)$ from $x$ to $y$ in $r$ to be $\left\|p_{\text {opt }}^{r}(x, y)\right\|$.

In particular, if a straight-line path $\overline{x y}$ lies on a boundary edge $e$, we may consider that the path is inside either of the two regions $r$ and $r^{\prime}$ incident to $e$, and consequently, the distance between $x$ and $y$, i.e., $d_{e}(x, y)$, is defined to be $\min \left\{d_{r}(x, y), d_{r^{\prime}}(x, y)\right\}$.

We say that $r$ is a pseudo-Euclidean region if it satisfies the following two properties.

Property 2: The function $d_{r}:\left(\mathcal{R}^{2}, \mathcal{R}^{2}\right) \rightarrow \mathcal{R}^{+} \bigcup\{0\}$ induced by the path cost metric of $r$ has the property that, for any two points $x, y \in r$ :

2.a) $d_{r}(x, y)=0$ if and only if $x=y$;

2.b) for any path $p(x, y)$ connecting $x$ and $y,\|p(x, y)\|=$ $d_{r}(x, y)$ if and only if $p$ is a straight-line segment ${ }^{2}$;

2.c) $d_{r}(x, y)$ can be computed in constant time given all geometric parameters of the region $r$.

Property 3: For any point $x_{0}$ in region $r$ (including the boundary) and any boundary edge $e=\overline{v_{0} v_{1}}$ of $r$ that is not incident to $x_{0}$, the number of local extrema for function $g_{x_{0}, e}$ : $[0,1] \rightarrow \mathcal{R}^{+}$is bounded by a constant, where $g_{x_{0}, e}(\lambda) \equiv$ $d_{r}\left(x_{0}(1-\lambda) v_{0}+\lambda v_{1}\right)$. Moreover, these local extrema can be computed efficiently.

Here, $\mathcal{R}$ denotes the reals and $\mathcal{R}^{+}$denotes the positive reals.

\footnotetext{
${ }^{2}$ This is not always necessary. See discussion in the following subsection on the anisotropic optimal path problem.
}

It is important to note that, although the properties stated above seem to be restrictive, they are satisfied by the cost metrics of many known path planning problems. Indeed, if the space can be divided into polygonal regions in each of which the cost metric can be uniformly defined, the subpath of an optimal path is usually straight inside each region (or can be replaced by an alternative straight-line subpath).

A space is said to be a piecewise pseudo-Euclidean space if it consists of a finite number of pseudo-Euclidean regions. Further, for an arbitrary path $p$ in space, if $p$ can be decomposed into subpaths $p\left[v_{1}, v_{2}\right], p\left[v_{2}, v_{3}\right], \ldots, p\left[v_{m-1}, v_{m}\right]$ such that each subpath $p\left[v_{i}, v_{i+1}\right], 1 \leq i<m$, lies entirely inside a region $r_{i}$, the cost $\|p\|$ of $p$ is defined to be $\sum_{i=1}^{m-1}\left\|p\left[v_{i}, v_{i+1}\right]\right\|$, which is the sum of the costs of all subpaths, each of which is determined by the path cost metric of the respective region. A piecewise pseudo-Euclidean optimal path problem is to find an optimal path (i.e., the path with the least cost) that joins two vertices $s$ and $t$ in a piecewise pseudo-Euclidean space. In Section II-D, we will give some examples of piecewise pseudoEuclidean spaces.

Property 2.b states that a path $p$ in $r$ is facewise optimal only if $p$ is a straight-line segment. This is not necessarily true for certain problems (e.g., the anisotropic optimal path problem). This property can be replaced by a weaker but less intuitive one.

Property 4 (Alternative of Property 2.b): For any four points $x_{1}, y_{1}, x_{2}, y_{2} \in r, d_{r}\left(x_{1}, y_{1}\right)+d_{r}\left(x_{2}, y_{2}\right)>d_{r}\left(x_{1}, y_{2}\right)+$ $d_{r}\left(x_{2}, y_{1}\right)$ if line segments $\overline{x_{1} y_{1}}$ and $\overline{x_{2} y_{2}}$ intersect in the interior of $r .^{3}$

Either of Properties 2.b and 4 can guarantee that the lemma in [9, Lemma 1] still holds for a piecewise pseudo-Euclidean space. We reestablish the lemma as the following.

Lemma 1: In a piecewise pseudo-Euclidean space, any two optimal paths with the same source point cannot intersect in the interior of any region.

At a later point, we will explain the importance of Property 3 to our algorithm; we will also show how "efficient" the computation of local extrema needs to be.

To construct an edge-subdivision-based discretization of an instance of a piecewise pseudo-Euclidean optimal path problem, for any two discrete points $v_{1}$ and $v_{2}$ on the border of the same region $r$ (but not on the same boundary edge), each graph edge $\left(v_{1}, v_{2}\right)$ corresponds to a facewise optimal path $p_{\mathrm{opt}}^{r}\left(v_{1}, v_{2}\right)$ from $v_{1}$ to $v_{2}$ and is assigned a weight $w\left(v_{1}, v_{2}\right)=$ $d_{r}\left(v_{1}, v_{2}\right)$; however, if $v_{1}$ and $v_{2}$ are on the same boundary edge $e$, the weight $w\left(v_{1}, v_{2}\right)$ is defined to be $d_{e}\left(v_{1}, v_{2}\right)$.

\section{Modifications}

In the following, we show how to make modifications to the original BUSHWHACK algorithm so that it can compute

\footnotetext{
${ }^{3}$ We require that $d_{r}\left(x_{1}, y_{1}\right)+d_{r}\left(x_{2}, y_{2}\right)>d_{r}\left(x_{1}, y_{2}\right)+d_{r}\left(x_{2}, y_{1}\right)$ for the convenience of discussion. As a matter of fact, this assumption can be relaxed to $d_{r}\left(x_{1}, y_{1}\right)+d_{r}\left(x_{2}, y_{2}\right) \geq d_{r}\left(x_{1}, y_{2}\right)+d_{r}\left(x_{2}, y_{1}\right)$. In this case, there may be two optimal paths intersecting inside a region; however, these intersecting optimal paths can be replaced by two nonintersecting ones that are equally optimal.
} 
approximate optimal paths for all piecewise pseudo-Euclidean optimal path problems.

BUSHWHACK propagates optimal path information in a "lazy" and "best-first" manner. Once a discrete point $v$ on boundary edge $e$ is discovered, unlike Dijkstra's algorithm, BUSHWHACK does not simultaneously propagate the optimal discrete path $p_{\text {opt }}^{\prime}(s, v)$ to all discrete points in $I_{v, e, e^{\prime}}$. By propagation, we mean that for each point $v^{*} \in I_{v, e, e^{\prime}}$, a candidate optimal path $p_{\text {opt }}^{\prime}(s, v)+\overline{v v^{*}}$ is inserted into QLIST for future consideration as an optimal discrete path from $s$ to $v^{*}$. Instead, BUSHWHACK propagates to the discrete points in $I_{v, e, e^{\prime}}$ sequentially according to their region distances to $v$ (in increasing order). A candidate optimal path $p_{\mathrm{opt}}^{\prime}(s, v)+\overline{v v^{*}}$ is not inserted into QLIST until $p_{\text {opt }}^{\prime}(s, v)+\overline{v v_{\text {prev }}^{*}}$ is removed from QLIST, where $v_{\text {prev }}^{*}$ is the discrete point preceding $v$ in $I_{v, e, e^{\prime}}$.

The reason is that intervals are dynamic: a discrete point $v^{*}$ originally included in interval $I_{v, e, e^{\prime}}$ may "switch" to another interval $I_{v^{\prime}, e, e^{\prime}}$ once $v^{\prime}$ is discovered, which makes the insertion of candidate optimal path $p_{\mathrm{opt}}^{\prime}(s, v)+\overline{v v^{*}}$ into QLIST meaningless (as this path is clearly nonoptimal according to Definition 1). Therefore, the insertion of a candidate optimal path into QLIST should be postponed as much as possible.

1) Splitting Intervals Into Monotonic Intervals: To sequentially propagate $p_{\text {opt }}^{\prime}(s, v)$ to points in $I_{v, e, e^{\prime}}$, we need to sort these points according to the increasing order of the region distance from $v$. One approach is to further divide each interval into a number of monotonic intervals such that in each monotonic interval the points are readily sorted according to the region distance from $v$. For the weighted region optimal path problem, this is trivial as each interval can be divided into two monotonic intervals by the perpendicular point of $v$ on $e^{\prime}$.

For a general piecewise pseudo-Euclidean optimal path problem, according to Property 3 , the region distance function from $v$ to points on $e^{\prime}$ has a constant number of local extrema. Thus, $I_{v, e, e^{\prime}}$ can be divided into a constant number of parts by these local extrema so that the region distance from $v$ to points in each part is monotonically increasing or decreasing.

To divide a newly created interval $I_{v, e, e^{\prime}}$ into monotonic intervals, we need to compute the local extrema of the region distance function $g_{v, e^{\prime}}$ from $v$ to points on $e^{\prime}$. By Property 3 , for any pseudo-Euclidean region, these local extrema can be computed "efficiently." Here, we specify how efficient the computation needs to be. Since only one splitting is performed for each interval, as long as the cost of computing local extrema (and thus the cost of splitting) for each interval does not exceed $O(\ln m)$, the total cost of splitting will be bounded by $O(n m \ln m)$ and will not affect the total complexity of $O(n m \ln n m)$.

It should be noted that we only need to find among $m$ discrete values (corresponding to $m$ Steiner points) the values closest to the local extrema. It is easy to see that, as long as $g_{v, e}$ itself is a constant degree polynomial function or computing the local extrema of $g_{v, e}$ can be converted to computing the roots of a constant degree polynomial function, the local extrema of $g_{v, e}$ can be computed in $O(\ln m)$ time.

2) Propagating Interval Paths: We let $e$ and $e^{\prime}$ be two boundary edges of region $r$. Suppose interval $I_{v, e, e^{\prime}}$ contains

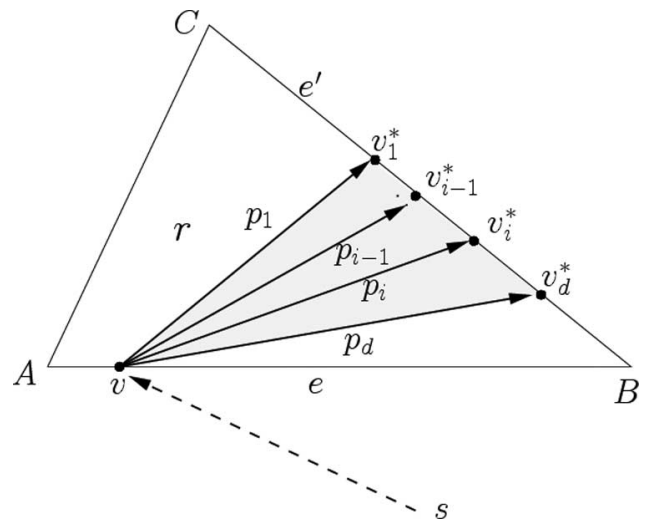

Fig. 2. Propagating interval paths.

points $v_{1}^{*}, v_{2}^{*}, \ldots, v_{d}^{*}$ when it is initially created (i.e., when $v$ is discovered), as shown in Fig. 2. Here, $v_{1}^{*}, v_{2}^{*}, \ldots, v_{d}^{*}$ are contiguous discrete points on $e^{\prime}$, and $v_{1}^{*}$ and $v_{d}^{*}$ are the two endpoints of the interval. Without loss of generality, we assume $d_{r}\left(v, v_{1}^{*}\right) \leq d_{r}\left(v, v_{d}^{*}\right)$. Since the interval $I_{v, e, e^{\prime}}$ is monotonic, we have $d_{r}\left(v, v_{1}^{*}\right) \leq d_{r}\left(v, v_{2}^{*}\right) \leq \cdots \leq d_{r}\left(v, v_{d}^{*}\right)$.

Let $p_{1}, p_{2}, \ldots, p_{d}$ be the direct interval paths associated with $I_{v, e, e^{\prime}}$, where $p_{i}=p_{\text {opt }}^{\prime}(s, v)+\overline{v v_{i}^{*}}$ for $1 \leq i \leq d$. For each $v_{i}^{*}$, let $P_{i}=\left\{p_{j, i} \mid 1 \leq j \leq d\right\}$, where $p_{j, i}=p_{j}+\overline{v_{j}^{*} v_{i}^{*}}$.

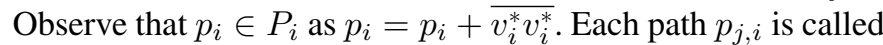
an extended interval path if $j \neq i$, as it extends a direct interval

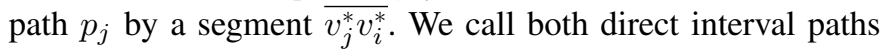
and extended interval paths interval paths. $P_{i}$ is the set of all interval paths associated with $I_{v, e, e^{\prime}}$ that connect $s$ and $v_{i}^{*}$. An interval path $p^{\prime} \in P_{i}$ is said to be locally optimal if $\left\|p^{\prime}\right\|=\min \left\{\|p\| \mid p \in P_{i}\right\}$.

Obviously, it is only necessary to insert those locally optimal paths into QLIST. For each $v_{i}^{*}$, BUSHWHACK inserts into QLIST only one locally optimal interval path $p_{i}^{*}$, which is called an adopted interval path, that connects $s$ and $v_{i}^{*}$. For the weighted region optimal path problem, BUSHWHACK [9] uses a simple scheme to compute the adopted interval paths efficiently by exploiting a geometric property of weighted regions induced by Snell's law. However, the scheme does not apply for general piecewise pseudo-Euclidean optimal path problems.

In the following, we will first show for a general piecewise pseudo-Euclidean optimal path problem how the BUSHWHACK algorithm determines adopted interval paths $p_{1}^{*}, p_{2}^{*}, \ldots, p_{d}^{*}$ for $I_{v, e, e^{\prime}}$, and then prove that these paths are locally optimal. Initially, interval path $p_{1}^{*}=p_{1}$ is inserted into QLIST. Iteratively, an interval path $p_{i}^{*}$ from $s$ to $v_{i}^{*}$ is added into QLIST when the interval path $p_{i-1}^{*}$ from $s$ to $v_{i-1}^{*}$ is extracted from QLIST. $p_{i}^{*}$ is chosen to be the less costly path between two paths $p_{i}$ and $p_{i-1}^{*}+\overline{v_{i-1}^{*} v_{i}^{*}}$.

That is, the interval path for $v_{i}^{*}$ is constructed by either:

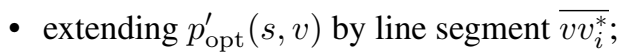

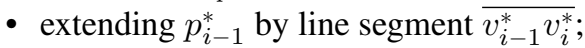

whichever is less costly. The propagation process terminates when all points in $I_{v, e, e^{\prime}}$ are reached by such interval paths. We can establish the following lemma. 
This article has been accepted for publication in a future issue of this journal, but has not been fully edited. Content may change prior to final publication.

Lemma 2: Each $p_{i}^{*}$ is locally optimal.

Proof: The lemma is proved by induction.

Basis Step:) Since each interval is monotonic, we have $d_{r}\left(v, v_{1}^{*}\right) \leq d_{r}\left(v, v_{2}^{*}\right) \leq \cdots \leq d_{r}\left(v, v_{d}^{*}\right)$ and thus the following inequality holds:

$$
\begin{aligned}
\left\|p_{1}^{*}\right\| & =\left\|p_{\mathrm{opt}}^{\prime}(s, v)\right\|+d_{r}\left(v, v_{1}^{*}\right) \\
& \leq\left\|p_{\mathrm{opt}}^{\prime}(s, v)\right\|+d_{r}\left(v, v_{j}^{*}\right) \\
& <\left\|p_{\mathrm{opt}}^{\prime}(s, v)\right\|+d_{r}\left(v, v_{j}^{*}\right)+d_{e^{\prime}}\left(v_{j}^{*}, v_{1}^{*}\right) \\
& =\left\|p_{j, 1}\right\| .
\end{aligned}
$$

Therefore, $p_{1}^{*}$ is locally optimal according to the definition.

Inductive Step:) Suppose $p_{i-1}^{*}$ is locally optimal. According to the definition of $p_{i}^{*}$, we have $\left\|p_{i}^{*}\right\|=$ $\min \left\{\left\|p_{i}\right\|,\left\|p_{i-1}^{*}+\overline{v_{i-1}^{*} v_{i}^{*}}\right\|\right\}$.

1) For each $j \geq i$ : $\left\|p_{i}^{*}\right\| \leq\left\|p_{i}\right\| \leq\left\|p_{j}\right\|<$ $\left\|p_{j, i}\right\|$.

2) For each $j<i$ : with the induction hypothesis, we have the following inequality:

$$
\begin{aligned}
\left\|p_{j, i}\right\|= & \left\|p_{j}\right\|+d_{e^{\prime}}\left(v_{j}^{*}, v_{i}^{*}\right) \\
= & \left\|p_{j}\right\|+d_{e^{\prime}}\left(v_{j}^{*}, v_{j+1}^{*}\right)+d_{e^{\prime}}\left(v_{j+1}^{*}, v_{j+2}^{*}\right)+ \\
& \cdots+d_{e^{\prime}}\left(v_{i-1}^{*}, v_{i}^{*}\right) \\
\geq & \left\|p_{j+1}^{*}\right\|+d_{e^{\prime}}\left(v_{j+1}^{*}, v_{j+2}^{*}\right)+\cdots+d_{e^{\prime}}\left(v_{i-1}^{*}, v_{i}^{*}\right) \\
& \vdots \\
\geq & \left\|p_{i-1}^{*}\right\|+d_{e^{\prime}}\left(v_{i-1}^{*}, v_{i}^{*}\right) \\
\geq & \left\|p_{i}^{*}\right\|
\end{aligned}
$$

Therefore, $p_{i}^{*}$ is also locally optimal.

This completes the proof.

\section{Known Piecewise Pseudo-Euclidean Optimal Path Problems}

In the following, we describe three problems that belong to the class of piecewise pseudo-Euclidean optimal path problems and have been investigated recently.

1) Weighted Region Optimal Path Problem: Clearly, this problem is a relatively simple problem of this class. Although regions in space are assigned with different unit weights, inside each region it is still a Euclidean space, and thus the straight-line path is facewise optimal (satisfying Property 2.b). To compute monotonic intervals, we simply need to divide each interval $I_{v, e, e^{\prime}}$ by the perpendicular point $v_{h}$ of $v$ on $e^{\prime}$ (satisfying Property 3 ).

2) Flow Path Problem: This problem is a complex generalization of the weighted region optimal path problem, which introduces inside each region a uniform flow that could affect the motion of the robot. The goal is to compute the least time consuming path between two given points. In this case, a facewise optimal path between two points is still the straightline path (satisfying Property 2.b). The region distance function from $v$ to any boundary edge $e^{\prime}$ only has one local extremum, which is computable in constant time by solving a linear equation (satisfying Property 3 ). Therefore, this problem is also a piecewise pseudo-Euclidean optimal path problem.

3) Anisotropic Optimal Path Problem: Due to the special (angular) ranges of traversal directions caused by power limitations or overturn danger, a straight-line path from $v_{1}$ to $v_{2}$ may be considered as impermissible if the traversal direction is inside one of those ranges, and thus, all facewise optimal paths from $v_{1}$ to $v_{2}$ are zigzag paths. Therefore, the cost metric of this problem does not satisfy Property 2.b. However, it does satisfy the weaker Property 4. Further, the region distance function from $v$ to any boundary edge $e^{\prime}$ also has only one local extremum: it is either the perpendicular point $v_{h}$ of $v$ on $e^{\prime}$ or one of the two points where the two rays bounding the special range containing $v_{h}$ intersect with $e^{\prime}$ and thus is computable in constant time as well (satisfying Property 3 ).

In addition, BUSHWHACK can also accommodate other variants of path cost metrics such as "crossing cost" for a path to pass through a boundary edge $e$ as long as $e$ can be divided into a constant number of segments such that the crossing cost is uniform inside each segment (so that the correctness of BUSHWHACK, or more specifically the interval path propagation scheme, can be guaranteed).

\section{Adaptive Discretization Method}

\section{A. Motivation}

The size of $\mathcal{G}_{\epsilon}$ can be very large even for a modest $\epsilon$ as the number of Steiner points placed on each boundary edge is also determined by a number of geometric parameters (see [10], [11], and [16]). Therefore, computing an $\epsilon$-good approximate optimal path by directly applying a discrete search algorithm to $\mathcal{G}_{\epsilon}$ may be very costly. In particular, a discrete search algorithm such as Dijkstra's algorithm or BUSHWHACK will compute an optimal discrete path from $s$ to every point $v \in \mathcal{G}_{\epsilon}$ that is closer to $s$ than $t$ is, which means that it has to search through a large space with the same (small) error tolerance $\epsilon$.

Here, we further elaborate the flight ticket booking example. With the knowledge accumulated through past experiences, the travel agent may know, for any intermediate airport A, a lower bound $L_{H, A}$ of the cost of a direct flight from Hong Kong to A as well as a lower bound $L_{A, S}$ of the cost of a direct flight from A to San Francisco. Further, she also knows an upper bound, for example, $\$ 1300$, of the cost of the cheapest flight (with one stop) from Hong Kong to San Francisco. In that case, the travel agent would only consider airport $\mathrm{A}$ as a possible stop between Hong Kong and San Francisco if $L_{H, A}+L_{A, S}<$ 1300. For example, it is at least worth the effort to check the ticketing database to find out the exact cost of the flight from Hong Kong to San Francisco via Shanghai as $1050+200<$ 1300 (see Fig. 3).

For shortest path problems, the $\mathrm{A}^{*}$ algorithm partially addresses this issue as it would first explore points that are estimated using a heuristic function to be closer to the destination point $t$. However, if the cost metric varies significantly in different regions (e.g., for the weighted region optimal path 


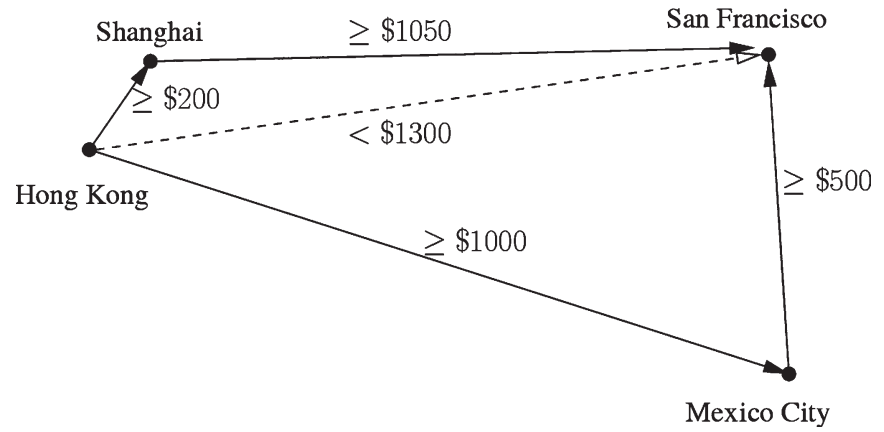

Fig. 3. Searching for the cheapest flight.

problem, the ratio between the maximum and minimum unit weights is large), it is difficult to find a heuristic function that can provide an accurate estimation of the distance (according to the defined cost metric) between a given point and $t$. As a result, the $\mathrm{A}^{*}$ algorithm may still have to search through many points in $\mathcal{G}_{\epsilon}$ unnecessarily. ${ }^{4}$

\section{B. Approximating in Multiple Rounds}

Here, we introduce a multiround approximation algorithm that uses an adaptive discretization method. For each $i, 1 \leq i \leq d$; this method computes an $\epsilon_{i}$-good approximate path from $s$ to $t$ in a subgraph $\mathcal{G}_{\epsilon_{i}}^{\prime}$ of $\mathcal{G}_{\epsilon_{i}}$, where $\epsilon_{1}>\epsilon_{2}>\cdots>$ $\epsilon_{d-1}>\epsilon_{d}=\epsilon$. (Recall that for a given discretization scheme (e.g., [10]), the discretization, and hence the number of Steiner points used, is fixed only for a given $\epsilon$.) In each round, with the approximate optimal path information acquired through the previous round, the algorithm can identify for each boundary edge the portion of the edge where more Steiner points need to be placed to guarantee an approximate optimal path with a reduced error bound. For the rest of the boundary edge, no further Steiner point needs to be placed.

For a given discretization, we call a segment of a boundary edge bounded by two adjacent discrete points a Steiner segment. A path $p^{\prime}$ is said to be neighboring an optimal path $p_{\text {opt }}$ if, for any Steiner segment that $p_{\text {opt }}$ crosses, $p^{\prime}$ passes through one of the two discrete points that bound the Steiner segment. Fig. 4 shows an optimal path and its neighboring discrete path.

The adaptive discretization method is a generic method applicable to many approximation algorithms for various optimal path problems. The only prerequisite is that the discretization scheme used for the approximation algorithm satisfies the following property.

1) Property 5: For any optimal path $p_{\text {opt }}$ connecting any two vertices $v_{1}$ and $v_{2}$ in the original (continuous) space, there is a neighboring discrete path $p\left(v_{1}, v_{2}\right)$ from $v_{1}$ to $v_{2}$ in the discretization with a cost no more than $(1+\epsilon) \cdot\left\|p_{\text {opt }}\left(v_{1}, v_{2}\right)\right\|$.

This discretization method can be applied to the weighted region optimal path problem as Property 5 holds for the discretization schemes in [7] and [10] and the one with reduced size [9]. It can also be applied to the flow path problem [11], the anisotropic optimal path problem [15], [16], and

\footnotetext{
${ }^{4}$ This is the case even when bidirectional search is adopted, which can reduce the search space by only a constant factor.
}

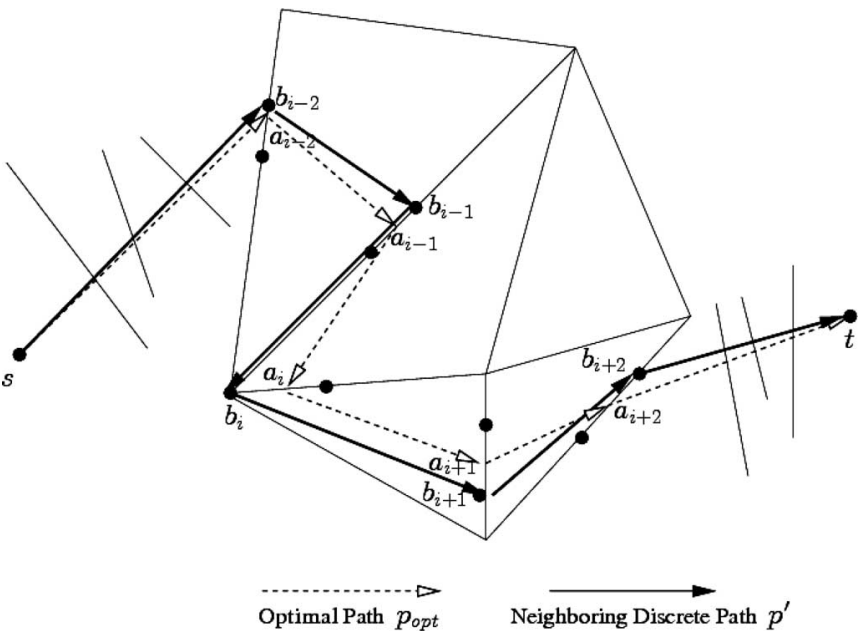

Fig. 4. Optimal path $p_{\text {opt }}$ crosses region boundaries at points $a_{1}, a_{2}, \ldots, a_{i-1}, a_{i}, \ldots$, while the neighboring discrete path $p^{\prime}$ crosses region boundaries at Steiner points $b_{1}, b_{2}, \ldots, b_{i-1}, b_{i}, \ldots$

the 3-D Euclidean shortest path problem [19], [20], as these problems all have discretization schemes suitable for adaptive discretization.

For any $i$, we denote the optimal discrete path found between any two points $v_{1}, v_{2} \in \mathcal{G}_{\epsilon_{i}}^{\prime}$ by $p_{\epsilon_{i}}^{\prime}\left(v_{1}, v_{2}\right)$. In the $i$ th round, a forward search is conducted to compute the optimal discrete path $p_{\epsilon_{i}}^{\prime}(s, v)$ from $s$ to every point $v \in \mathcal{G}_{\epsilon_{i}}^{\prime}$ until the cost of such a path grows beyond $\left(1+\epsilon_{i}\right) \cdot\left\|p_{\epsilon_{i}}^{\prime}(s, t)\right\|$. We call each of these points a searched point. A backward search is then conducted to compute the optimal discrete path $p_{\epsilon_{i}}^{\prime}(v, t)$ from every searched point $v$ to $t$.

We call a point $v$ a useful point if either $\left\|p_{\epsilon_{i}}^{\prime}(s, v)\right\|+$ $\left\|p_{\epsilon_{i}}^{\prime}(v, t)\right\| \leq\left(1+\epsilon_{i}\right) \cdot\left\|p_{\epsilon_{i}}^{\prime}(s, t)\right\|$ or $v$ is a vertex; we call a Steiner segment a useful segment if at least one of its endpoints is useful. Note that the smaller $\epsilon_{i}$ is, the more restrictive this condition is, and thus the stronger the pruning will be. We claim that (as proved in Theorem 1) an optimal path from $s$ to $t$ will not pass through a useless segment, and therefore, in the next round, we only need to further refine the discretization (by inserting more Steiner points) in the useful segments.

The details of the adaptive discretization method are shown in Algorithm 1. Note that the two searches (one forward and one backward) in each round may be performed simultaneously using Dijkstra's two-tree algorithm [21]-[23].

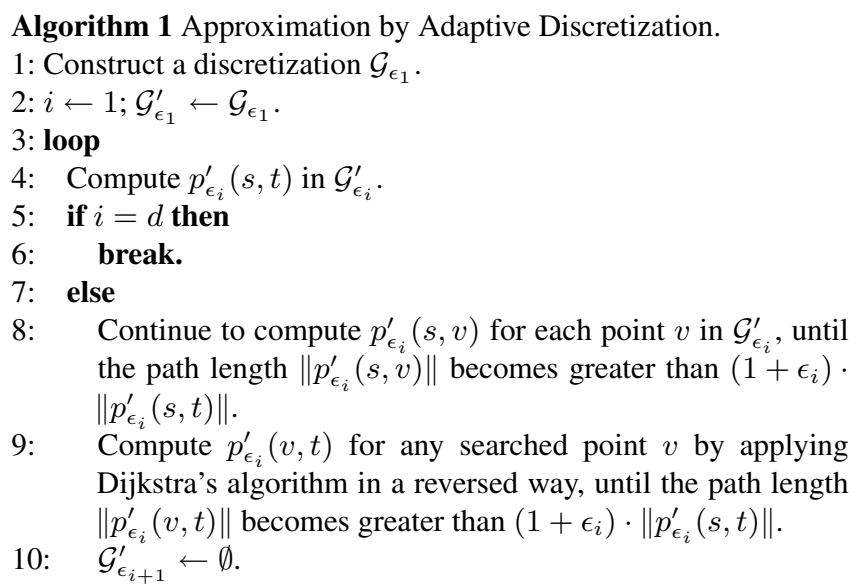

1: Construct a discretization $\mathcal{G}_{\epsilon_{1}}$

$2: i \leftarrow 1 ; \mathcal{G}_{\epsilon_{1}}^{\prime} \leftarrow \mathcal{G}_{\epsilon_{1}}$.

3: loop

4: Compute $p_{\epsilon_{i}}^{\prime}(s, t)$ in $\mathcal{G}_{\epsilon_{i}}^{\prime}$.

5: $\quad$ if $i=d$ then

6: break.

7: else

10. $\quad \mathcal{G}_{\epsilon_{i+1}}^{\prime} \leftarrow \emptyset$ 
11: for all useful point $v \in \mathcal{G}_{\epsilon_{i}}^{\prime}$ do

12: $\quad$ Add $v$ into $\mathcal{G}_{\epsilon_{i+1}}^{\prime}$.

13: for all point $v \in \mathcal{G}_{\epsilon_{i+1}}$ do

14: $\quad$ if $v$ is located inside a useful Steiner segment of $\mathcal{G}_{\epsilon_{i}}^{\prime}$ then

15: $\quad$ Add $v$ into $\mathcal{G}_{\epsilon_{i+1}}^{\prime}$.

16: $i \leftarrow i+1$.

17: return $p_{\epsilon_{i}}^{\prime}(s, t)$.

This discretization method has both pros and cons when compared against the fixed discretization method. It has to run a discrete search algorithm on $d$ different graphs, and each time, it involves both forward and backward searches (except for the last round, which involves only a forward search). However, in the earlier rounds, it explores approximate optimal paths with high error tolerance, while in later rounds, as it gradually reduces the error tolerance, it only searches approximate optimal paths in a small subspace (that is, the useful segments of the boundary edges) instead of the entire original space (all boundary edges). Our experimental results show that, when the desired error tolerance $\epsilon$ is small, the adaptive discretization method performs more efficiently than the fixed discretization assuming that for both methods the same discrete search algorithm is used.

\section{Performance Improvement: A Simple Illustration}

To provide an intuitive illustration of the advantage of the adaptive discretization method, we assume that the problem is, hypothetically, to find approximate optimal paths in 2-D space that contain regions with unit area and where the minimum cost from any two points is roughly proportional to the Euclidean distance between them. We further assume that the number of Steiner points placed in each region is $(1 / \epsilon) \ln (1 / \epsilon)$ for any given $\epsilon>0$.

To compute a (1/16)-good approximate optimal path using the fixed discretization method, when an optimal discrete path from $s$ to $t$ in $\mathcal{G}_{1 / 16}$ is determined, Dijkstra's algorithm has computed optimal discrete paths from $s$ to all points inside a circle with radius $R$ (roughly speaking), where $R$ is the Euclidean distance from $s$ to $t$ [see Fig. 5(a)]. Let $n$ be the number of regions inside this circle, and let $N=n \cdot 16 \ln 16=$ $44.3614 \cdot n$ denote the number of Steiner points inside the circle. The time spent is therefore $O(N \ln N)$.

Now, we instead use a two-round adaptive discretization method with $\epsilon_{1}=4 \cdot \epsilon=(1 / 4)$. The first (forward) search of Round 1 is to compute an optimal discrete path from $s$ to any point in $\mathcal{G}_{1 / 4}$ that is inside the circle with a radius of $5 R / 4$ [see Fig. 5(b)], which contains $25 \cdot n / 16$ regions and therefore $N_{1}=(25 \cdot n / 16) \cdot 4 \ln 4=8.6643 \cdot n$ Steiner points. The second (backward) search of Round 1 is to compute an optimal path from $v$ to $t$ for each discovered point $v$ in $\mathcal{G}_{1 / 4}$ such that $|\overline{v s}|+|\overline{v t}| \leq(5 R / 4)$. The number of such Steiner points is roughly $N_{2}=(15 \cdot n / 64) \cdot 4 \ln 4=1.2997 \cdot n$, as these points are bounded by an oval with a long axis of $5 R / 4$ and a short axis of $3 R / 4$ [see Fig. 5(c)].

The second round involves computing an optimal discrete path from $s$ to each point $v$ in $\mathcal{G}_{1 / 16}$ that is inside the same oval and within a distance of $R$ from $s$ [see Fig. 5(d)]. The number

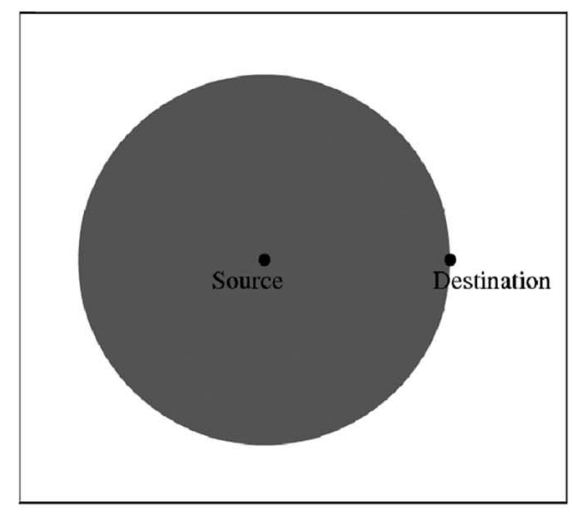

(a)

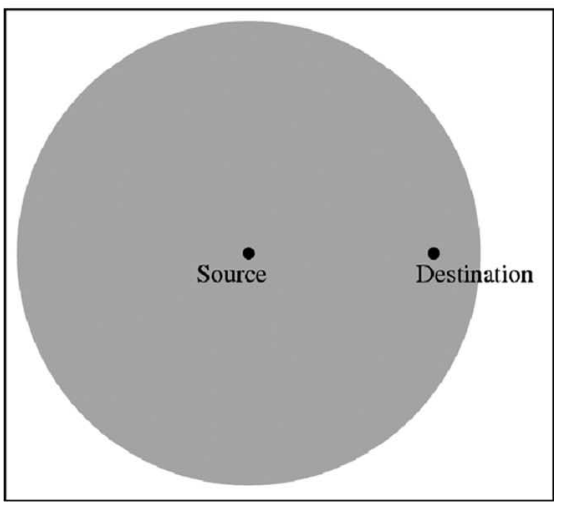

(b)

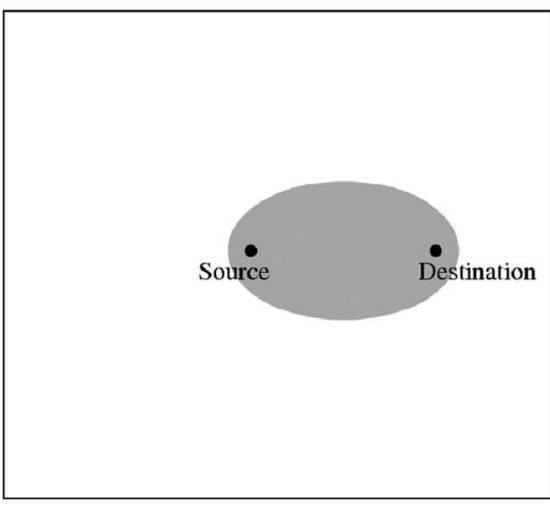

(c)

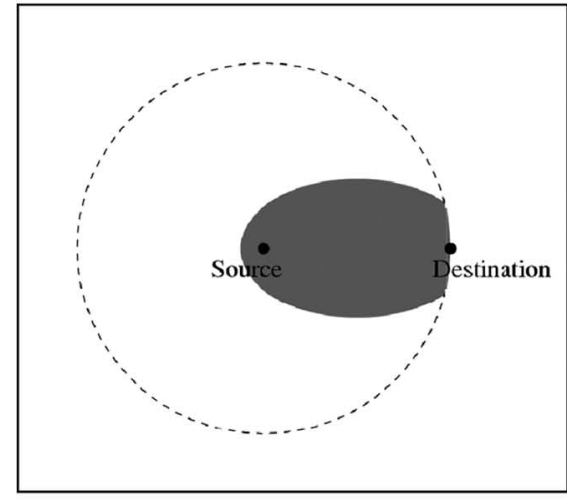

(d)

Fig. 5. Comparison on search space by fixed and adaptive discretization methods. (a) Fixed discretization. (b) Adaptive discretization, Round 1, forward search. (c) Adaptive discretization, Round 1, backward search. (d) Adaptive discretization, Round 2, forward search. 
of Steiner points "touched" in the second round is bounded by $N_{3}=(15 \cdot n / 64) \cdot 16 \ln 16=10.3972 \cdot n$. Therefore, the total number of Steiner points used by running this two-round adaptive discretization method is $N_{1}+N_{2}+N_{3}=20.3612 \cdot n$.

The speedup ratio therefore is

$$
\frac{N \ln N}{\sum_{i=1}^{3} N_{i} \ln N_{i}}>\frac{N}{\sum_{i=1}^{3} N_{i}}=\frac{44.3614 \cdot n}{20.3612 \cdot n}=2.1787
$$

It is important to note that the actual speedup ratio may be greater than what we have analyzed above as the computation time required is not determined entirely by $O(N \ln N)$, where again $N$ is the number of Steiner points. For Dijkstra's algorithm, $O(N \ln N)$ reflects only the cost of extracting the least costly path $N$ times (one for each discovered point) from the path list QLIST [a priority list of size $O(N)$ ] but not the cost of updating neighboring points once an optimal discrete path is determined. The latter part has a higher dependency on $1 / \epsilon$. With adaptive discretization, each time the least costly path is extracted from QLIST (except in the last round), fewer neighbors need to be updated as the graph is sparser than $\mathcal{G}_{\epsilon}$.

\section{Correctness Proof}

We still need to prove the correctness of our multiround approximation algorithm. It suffices to show the following theorem.

Theorem 1: For any optimal path $p_{\text {opt }}(s, t)$, in each $\mathcal{G}_{\epsilon_{i}}^{\prime}$, there is a neighboring discrete path $p^{\prime}(s, t)$ with a cost no more than $\left(1+\epsilon_{i}\right) \cdot\left\|p_{\text {opt }}(s, t)\right\|$.

Proof: The theorem is proved by induction.

Basic Step: When $i=1, \mathcal{G}_{\epsilon_{i}}^{\prime}=\mathcal{G}_{\epsilon_{1}}$, and therefore the proposition is true, according to Property 5.

Inductive Step: We assume that, for any optimal path $p_{\text {opt }}(s, t), \mathcal{G}_{\epsilon_{i}}^{\prime}$ contains a discrete path $p^{\prime}(s, t)$ neighboring $p_{\text {opt }}(s, t)$ such that $\left\|p^{\prime}(s, t)\right\| \leq\left(1+\epsilon_{i}\right) \cdot\left\|p_{\text {opt }}(s, t)\right\|$.

We first show that $p_{\text {opt }}(s, t)$ will not pass through any useless Steiner segment in $\mathcal{G}_{\epsilon}^{\prime}$. Suppose otherwise that $p_{\text {opt }}(s, t)$ passes through a point inside a useless segment $\overline{u_{1} u_{2}}$, as shown in Fig. 6. According to the induction hypothesis, we can construct a neighboring discrete path $p^{\prime}(s, t)$ from $s$ to $t$ such that $\left\|p^{\prime}(s, t)\right\| \leq\left(1+\epsilon_{i}\right) \cdot\left\|p_{\text {opt }}(s, t)\right\|$. This implies that $p^{\prime}(s, t)$ passes through either $u_{1}$ or $u_{2}$. Without loss of generality, we assume that $p^{\prime}(s, t)$ passes through $u_{1}$. Since $u_{1}$ is useless, we have $\left\|p_{\epsilon_{i}}^{\prime}\left(s, u_{1}\right)\right\|+\left\|p_{\epsilon_{i}}^{\prime}\left(u_{1}, t\right)\right\|>\left(1+\epsilon_{i}\right) \cdot\left\|p_{\epsilon_{i}}^{\prime}(s, t)\right\| \geq$ $\left(1+\epsilon_{i}\right) \cdot\left\|p_{\text {opt }}(s, t)\right\| \geq\left\|p^{\prime}(s, t)\right\|$, which is a contradiction to the fact that $p^{\prime}(s, t)$ cannot be less costly than the concatenation of $p_{\epsilon_{i}}^{\prime}\left(s, u_{1}\right)$ and $p_{\epsilon_{i}}^{\prime}\left(u_{1}, t\right)$.

Since any optimal path from $s$ to $t$ will not pass through a useless Steiner segment, $\mathcal{G}_{\epsilon_{i+1}}^{\prime}$, which includes all the Steiner points of $\mathcal{G}_{\epsilon_{i+1}}$ except those inside useless Steiner segments, contains every discrete path in $\mathcal{G}_{\epsilon_{i+1}}$ that neighbors one of the optimal paths from $s$ to $t$, which includes at least one with a cost no more than $\left(1+\epsilon_{i+1}\right) \cdot\left\|p_{\text {opt }}(s, t)\right\|$, as guaranteed by Property 5.

This completes the proof.

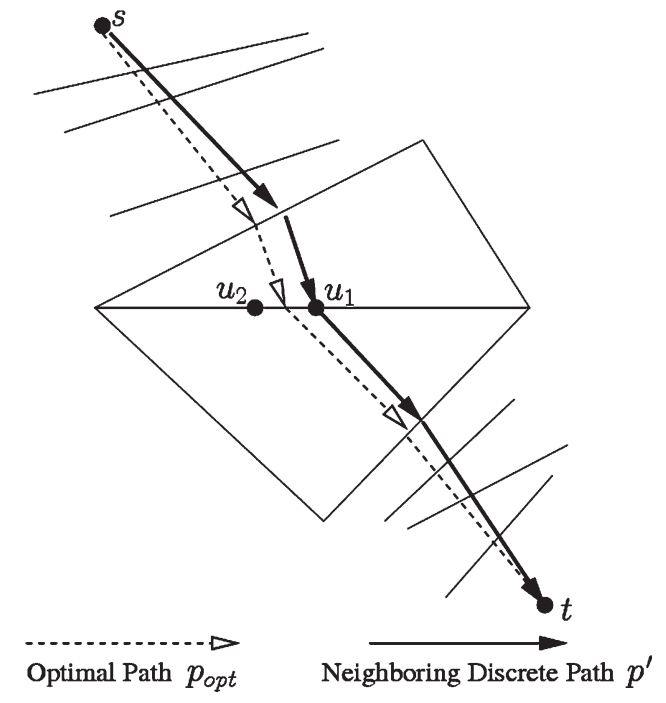

Fig. 6. Optimal path crossing a useless segment.

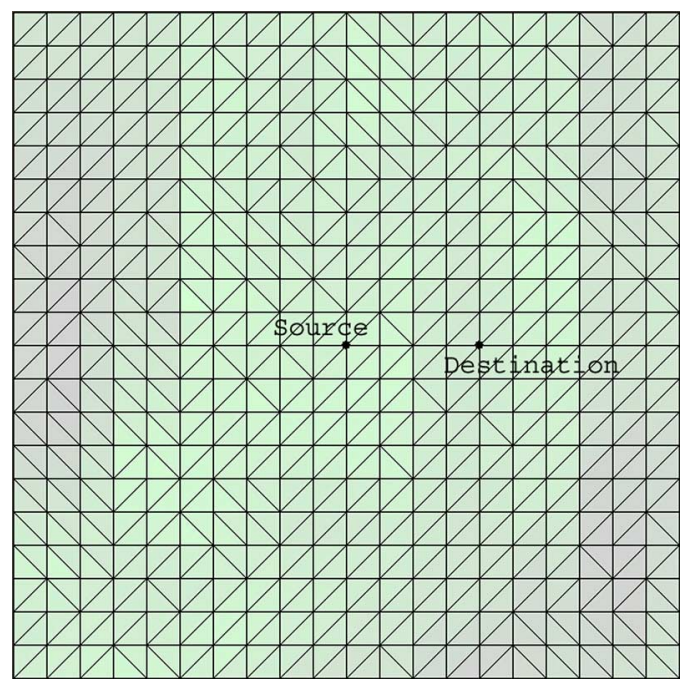

Fig. 7. TIN converted from $20 \times 20$ grid patch viewed from the top (with source and destination points picked).

\section{EXPERIMENTAL RESULTS}

In order to provide a performance comparison, we implemented using Java the following three algorithms:

- Algorithm 1: BUSHWHACK;

- Algorithm 2: pure Dijkstra's algorithm, which searches every incident edge of a discrete point in $\mathcal{G}_{\epsilon}$;

- Algorithm 3: two-round adaptive discretization method, which uses pure Dijkstra's algorithm for each search and chooses $\epsilon_{1}=3 \cdot \epsilon$.

For our experiments, we chose Triangular Irregular Networks, or TINs, converted from terrain maps in grid data format. More specifically, we used the digital elevation model file of Kaweah River basin. It is a $1424 \times 1163$ grid with $30 \mathrm{~m}$ between two neighboring grid points. We randomly took from the terrain map 30 patches: ten $20 \times 20$ ones, ten $40 \times 40$ ones, and ten $80 \times 80$ ones, respectively. Each patch was converted to a TIN by connecting two grid points diagonally for each grid cell. For each triangular face $r$, we assign to $r$ a unit weight $w_{r}$ 
TABLE I

PERformanCE Statistics

\begin{tabular}{|c|c|c|c|c|c|c|}
\hline $\begin{array}{l}\text { TIN } \\
\text { Size }\end{array}$ & $\frac{1}{\epsilon}$ & $\begin{array}{l}\text { Algo- } \\
\text { rithm }\end{array}$ & $\begin{array}{c}\text { Time } \\
(\mathrm{sec} .)\end{array}$ & $\begin{array}{c}\text { Number } \\
\text { Steiner } \\
\text { Points }\end{array}$ & $\begin{array}{c}\text { Number } \\
\text { Edges } \\
(\times 1000) \\
\end{array}$ & $\begin{array}{c}\text { Number } \\
\text { Discovered } \\
\text { Points } \\
\end{array}$ \\
\hline \multirow{15}{*}{$\begin{array}{c}20 \\
\times \\
20\end{array}$} & \multirow{3}{*}{3} & $\overline{1}$ & $\overline{3.7}$ & 18,233 & $\overline{306}$ & $\overline{\overline{13.050}}$ \\
\hline & & 2 & 5.4 & 18,233 & 2,353 & 13,050 \\
\hline & & 3 & 6.0 & 25,347 & 2,046 & 26,238 \\
\hline & \multirow{3}{*}{5} & 1 & 7.0 & 33,852 & 602 & 24,171 \\
\hline & & 2 & 16.5 & 33,852 & 7,946 & 24,171 \\
\hline & & 3 & 10.3 & 32,208 & 4,466 & 32,295 \\
\hline & \multirow{3}{*}{7} & 1 & 10.4 & 50,753 & 942 & 36,199 \\
\hline & & 2 & 35.9 & 50,753 & 17,698 & 36,199 \\
\hline & & 3 & 17.1 & 39,730 & 7,859 & 39,400 \\
\hline & \multirow{3}{*}{9} & 1 & 14.7 & 68,571 & 1,312 & 48,880 \\
\hline & & 2 & 64.8 & 68,571 & 32,141 & 48,880 \\
\hline & & 3 & 24.4 & 46,376 & 11,920 & 45,559 \\
\hline & \multirow{3}{*}{11} & 1 & 18.7 & 87,087 & 1,704 & 62,057 \\
\hline & & 2 & 104.8 & 87,087 & 51,663 & 62,057 \\
\hline & & 3 & 33.4 & 52,670 & 16,638 & 51,653 \\
\hline \multirow{15}{*}{$\begin{array}{c}40 \\
\times \\
40\end{array}$} & \multirow{3}{*}{3} & 1 & 24.2 & 89,907 & 1,838 & 77,059 \\
\hline & & 2 & 33.6 & 89,907 & 12,932 & 77,059 \\
\hline & & 3 & 30.3 & 112,951 & 10,014 & 123,442 \\
\hline & \multirow{3}{*}{5} & 1 & 43.0 & 166,882 & 3,621 & 142,826 \\
\hline & & 2 & 103.2 & 166,882 & 43,655 & 142,826 \\
\hline & & 3 & 53.2 & 144,410 & 20,909 & 152,086 \\
\hline & \multirow{3}{*}{7} & 1 & 66.4 & 250,212 & 5,660 & 214,034 \\
\hline & & 2 & 224.6 & 250,212 & 97,245 & 214,034 \\
\hline & & 3 & 82.1 & 175,565 & 35,000 & 180,076 \\
\hline & \multirow{3}{*}{9} & 1 & 92.3 & 338,056 & 7,901 & 289,111 \\
\hline & & 2 & 402.8 & 338,056 & 176,557 & 289,111 \\
\hline & & 3 & 120.9 & 206,805 & 53,440 & 210,513 \\
\hline & \multirow{3}{*}{11} & 1 & 118.2 & 429,442 & 10,345 & 367,137 \\
\hline & & 2 & 643.6 & 429,442 & 283,793 & 367,137 \\
\hline & & 3 & 167.0 & 239,613 & 75,656 & 242,058 \\
\hline \multirow{15}{*}{$\begin{array}{c}80 \\
\times \\
80\end{array}$} & \multirow{3}{*}{3} & 1 & 91.8 & 308,604 & 6,651 & 282,658 \\
\hline & & 2 & 130.0 & 308,604 & 47,232 & 282,658 \\
\hline & & 3 & 143.3 & 470,214 & 42,549 & 525,013 \\
\hline & \multirow{3}{*}{5} & 1 & 163.4 & 572,966 & 13,040 & 523,947 \\
\hline & & 2 & 407.0 & 572,966 & 159,375 & 523,947 \\
\hline & & 3 & 242.1 & 580,579 & 88,214 & 623,100 \\
\hline & \multirow{3}{*}{7} & 1 & 246.4 & 858,923 & 20,283 & 785,005 \\
\hline & & 2 & 873.9 & 858,923 & 354,750 & 785,005 \\
\hline & & 3 & 376.2 & 684,710 & 149,345 & 724,784 \\
\hline & \multirow{3}{*}{9} & 1 & 339.3 & $1,160,210$ & 28,230 & $1,060,062$ \\
\hline & & 2 & $1,563.1$ & $1,160,210$ & 643,629 & $1,060,062$ \\
\hline & & 3 & 540.3 & 788,757 & 224,506 & 826,978 \\
\hline & \multirow{3}{*}{11} & 1 & 445.3 & $1,473,380$ & 36,730 & $1,345,972$ \\
\hline & & 2 & $2,467.5$ & $1,473,380$ & $1,034,058$ & $1,345,972$ \\
\hline & & 3 & 748.2 & 895,747 & 315,104 & 933,344 \\
\hline
\end{tabular}

that is equal to $1+10 \tan \alpha_{r}$, where $\alpha_{r}$ is the absolute value of the angle between $r$ and the horizontal plane.

Note that the above formula for unit weight is chosen for the sole purpose of the experiments: the first part (constant 1) can be considered as the unit cost associated with friction, and the second part $\left(10 \tan \alpha_{r}\right)$ is the extra unit cost associated with traveling on a slope (whether uphill or downhill). ${ }^{5}$ The speedup ratios of both algorithms remain relatively stable when we pick other cost metrics (such as the one used for the flow path problem or the anisotropic optimal path problem).

For each TIN, we handpicked the source point with $x=0.5$ and $y=0.5$ as well as the destination point with $x=0.7$ and $y=0.5$ (assuming the projection of the terrain patch onto the

\footnotetext{
${ }^{5}$ In reality, a robot could gain energy by traveling downhill (while losing potential energy); however, for a pair of fixed source and destination points, the total potential energy gain (or loss) is a constant, and thus that part can be ignored.
}

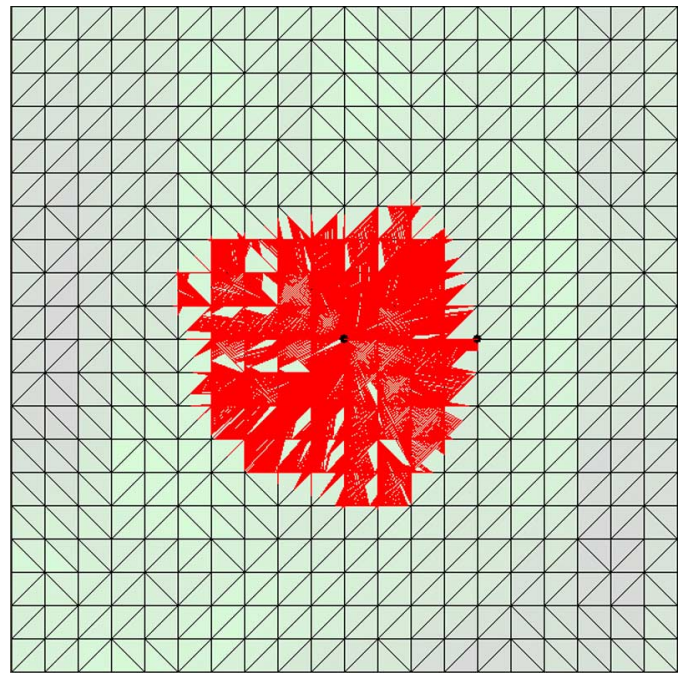

Fig. 8. Shortest path tree by fixed discretization method.

$x y$ plane is a unit square), as shown in Fig. 7. We used the logarithmic discretization scheme in [10] to generate the discretizations for all these algorithms. (Recall that for Algorithm 3, the discretization is acquired in multiple stages.) For each algorithm, we took the average of the running times of the experiments on all TINs with the same size. We repeated the experiments with $1 / \epsilon=3,5,7,9$, and 11 , respectively, and reported the results in Table I. All the timed results were acquired from a Sun Blade-1000 workstation with 4-GB memory. Note that the running times were acquired from a Java implementation. Therefore, the relative performance is more important than the absolute values of running times.

From Table I, it is easy to see that, when $\epsilon$ decreases, the running times of Algorithm 1 (BUSHWHACK algorithm) and Algorithm 3 (adaptive discretization method) are growing much slower than that of Algorithm 2 (pure Dijkstra's algorithm). We also listed the average number of visited edges for each algorithm and each value of $\epsilon$. It occurs to us that the number of visited edges and the running time are closely correlated.

We also listed in Table I the average number of Steiner points and the average number of discovered points for each experiment setting. In particular, for Algorithm 3, for the number of Steiner points, we will count a Steiner point twice if it is used in both rounds. Similarly, for the number of discovered points, a discrete point is counted as many times as it is discovered in both forward and backward searches in both rounds.

Algorithms 1 and 2 use the same number of Steiner points and also have the same number of discovered points, as they both use the fixed discretization method. For Algorithm 3, which has a larger $\epsilon$, it actually uses more Steiner points (collectively) and has more discovered points accordingly, as it has to search a large space in the first round. For example, with $\epsilon=1 / 3$, in the forward search of the first round, the algorithm has to compute optimal discrete paths to discrete points within a distance of $\left(1+\epsilon_{1}\right) \cdot\left\|p_{\epsilon_{1}}^{\prime}(s, t)\right\|=2 \cdot\left\|p_{\epsilon_{1}}^{\prime}(s, t)\right\| \geq$ $2 \cdot\left\|p_{\epsilon}^{\prime}(s, t)\right\|$ from $s$. The area covered by these discrete points is four times as large as the area covered by the discovered discrete point with the fixed discretization method [compare Figs. 8 and 9(a)]. Therefore, although Algorithm 3 searches the 


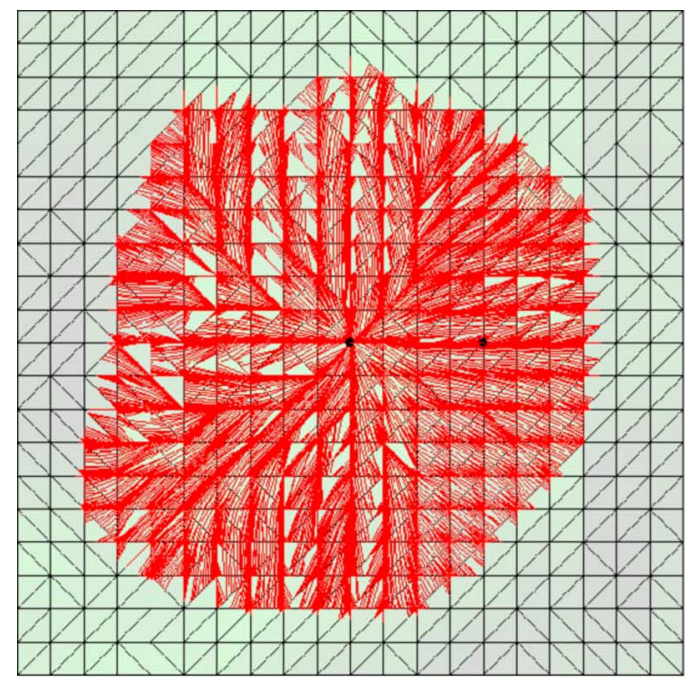

(a)

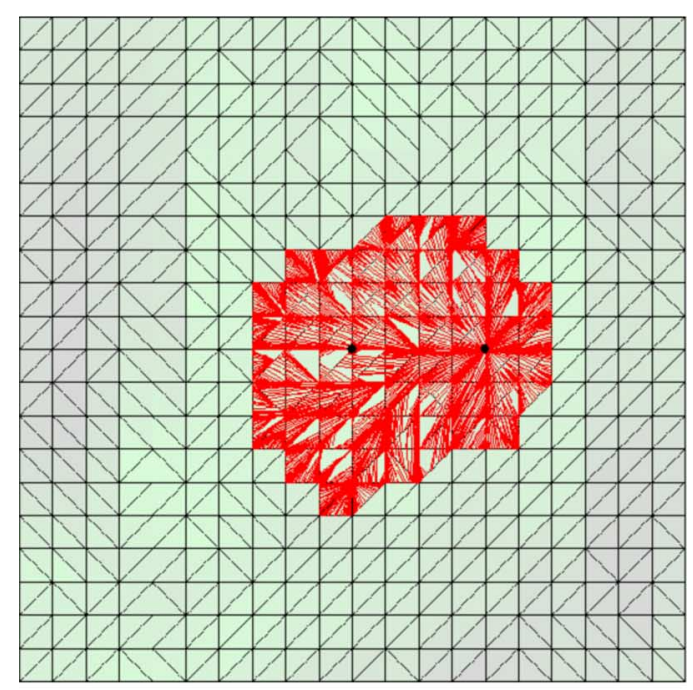

(b)

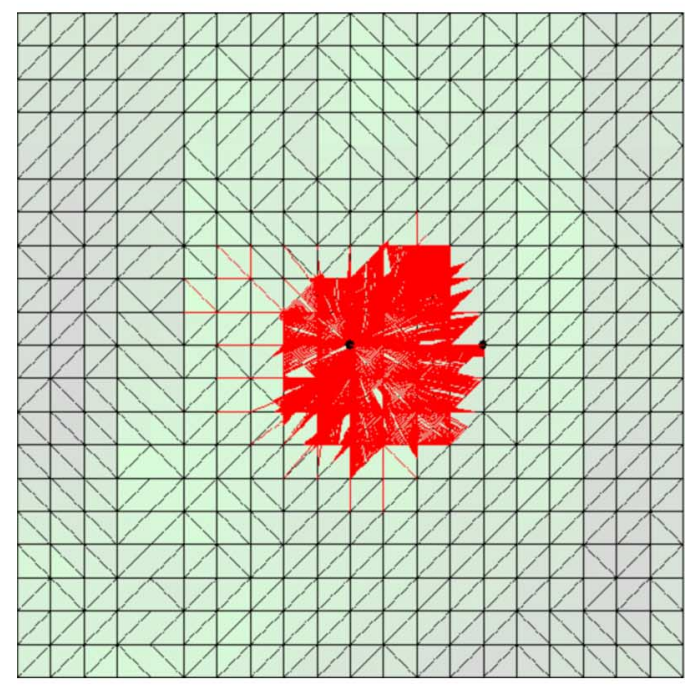

(c)

Fig. 9. Shortest path trees by adaptive discretization method. (a) First round, forward search. (b) First round, backward search. (c) Second round, forward search. space with less density of Steiner points in the first round, it still uses a large number of Steiner points. Similarly, because of the large $\epsilon_{1}$, the search space of the forward search of Algorithm 3 in the second round is just slightly smaller than that of the fixed discretization method [compare Figs. 8 and 9(c)] and so is the number of Steiner points saved.

However, as $\epsilon$ decreases, the saving on Steiner points by Algorithm 3 (as compared to Algorithms 1 and 2) becomes more and more significant and so is its speedup ratio (with respect to Algorithm 2), as indicated in Table I.

Note that here we did not experiment on combining the BUSHWHACK algorithm with the adaptive discretization method. Since BUSHWHACK already adopts its own pruning strategy, applying the adaptive discretization method to BUSHWHACK does not achieve as significant a speedup ratio as it does for the original Dijkstra's algorithm.

\section{CONCLUSION}

In this paper, we provided two results on the approximation algorithms for some robotic optimal path problems: 1) a generalization of the BUSHWHACK algorithm as well as a characterization of cost metrics for which the generalized BUSHWHACK algorithm can be applied; and 2) an adaptive discretization that selectively puts Steiner points with high density on boundary edges. It is important to note that, although in the paper we addressed the optimal path problems in 2-D spaces, all the results can be applied to polyhedral surfaces as well.

\section{ACKNOWLEDGMENT}

Part of the research was done when Z. Sun was at Duke University and Hong Kong Baptist University.

\section{REFERENCES}

[1] Z. Sun and J. H. Reif, "BUSHWHACK: An approximation algorithm for minimal paths through pseudo-Euclidean spaces," in Proc. 12th Annu. Int. Symp. Algorithms and Comput., 2001, vol. 2223, pp. 160-171.

[2] Z. Sun and J. H. Reif, "Adaptive and compact discretization for weighted region optimal path finding," in Proc. 14th Int. Symp. Fundam. Comput. Theory, 2003, vol. 2751, pp. 258-270.

[3] J. S. B. Mitchell, "Geometric shortest paths and network optimization," in Handbook of Computational Geometry, J.-R. Sack and J. Urrutia, Eds. Amsterdam, The Netherlands: Elsevier, 2000, pp. 633-701.

[4] J. S. B. Mitchell and C. H. Papadimitriou, "The weighted region problem: Finding shortest paths through a weighted planar subdivision," J. ACM, vol. 38, no. 1, pp. 18-73, Jan. 1991.

[5] C. Mata and J. S. B. Mitchell, "A new algorithm for computing shortest paths in weighted planar subdivisions," in Proc. 13th Annu. ACM Symp. Comput. Geom., 1997, pp. 264-273.

[6] M. Lanthier, A. Maheshwari, and J.-R. Sack, "Approximating weighted shortest paths on polyhedral surfaces," Algorithmica, vol. 30, no. 4, pp. 527-562, 2001 .

[7] L. Aleksandrov, M. Lanthier, A. Maheshwari, and J.-R. Sack, "An $\epsilon$ approximation algorithm for weighted shortest paths on polyhedral surfaces," in Proc. 6th Scandinavian Workshop Algorithm Theory, 1998, vol. 1432, pp. 11-22.

[8] L. Aleksandrov, A. Maheshwari, and J.-R. Sack, "Approximation algorithms for geometric shortest path problems," in Proc. 32nd Annu. ACM Symp. Theory Comput., 2000, pp. 286-295.

[9] Z. Sun and J. H. Reif, "On finding approximate optimal paths in weighted regions," J. Algorithms, vol. 58, no. 1, pp. 1-32, 2006.

[10] L. Aleksandrov, A. Maheshwari, and J.-R. Sack, "Determining approximate shortest paths on weighted polyhedral surfaces," J. ACM, vol. 52, no. 1, pp. 25-53, Jan. 2005. 
[11] J. H. Reif and Z. Sun, "Movement planning in the presence of flows," Algorithmica, vol. 39, no. 2, pp. 127-153, 2004.

[12] N. C. Rowe and R. S. Ross, "Optimal grid-free path planning across arbitrarily contoured terrain with anisotropic friction and gravity effects," IEEE Trans. Robot. Autom., vol. 6, no. 5, pp. 540-553, Oct. 1990.

[13] N. C. Rowe and Y. Kanayama, "Near-minimum-energy paths on a vertical-axis cone with anisotropic friction and gravity effects," Int. J. Robot. Res., vol. 13, no. 5, pp. 408-433, Oct. 1994.

[14] N. C. Rowe, "Obtaining optimal mobile-robot paths with non-smooth anisotropic cost functions using qualitative-state reasoning," Int. J. Robot. Res., vol. 16, no. 3, pp. 375-399, Jun. 1997.

[15] M. Lanthier, A. Maheshwari, and J.-R. Sack, "Shortest anisotropic paths on terrains," in Proc. 26th Int. Colloq. Automata, Languages and Program., 1999, vol. 1644, pp. 524-533.

[16] Z. Sun and J. H. Reif, "On finding energy-minimizing paths on terrains," IEEE Trans. Robot., vol. 21, no. 1, pp. 102-114, Feb. 2005.

[17] J. Hershberger and S. Suri, "An optimal algorithm for Euclidean shortest paths in the plane," SIAM J. Comput., vol. 28, no. 6, pp. 2215-2256, 1999.

[18] Z. Sun and T.-M. Bu, "On discretization methods for approximating optimal paths in regions with direction-dependent costs," Inf. Process. Lett., vol. 97 , no. 4, pp. 146-152, 2006.

[19] C. H. Papadimitriou, "An algorithm for shortest-path motion in three dimensions," Inf. Process. Lett., vol. 20, no. 5, pp. 259-263, 1985.

[20] J. Choi, J. Sellen, and C. K. Yap, "Approximate Euclidean shortest path in 3-space," in Proc. 10th Annu. ACM Symp. Comput. Geom., 1994, pp. $41-48$.

[21] G. B. Dantzig, "On the shortest route through a network," Manage. Sci., vol. 6, no. 2, pp. 187-190, Jan. 1960.

[22] T. A. J. Nicholson, "Finding the shortest route between two points in a network," Comput. J., vol. 9, no. 3, pp. 275-280, Nov. 1966.

[23] R. V. Helgason, J. Kennington, and B. Stewart, "The one-to-one shortestpath problem: An empirical analysis with the two-tree Dijkstra algorithm," Comput. Optim. Appl., vol. 2, no. 1, pp. 47-75, Jun. 1993.

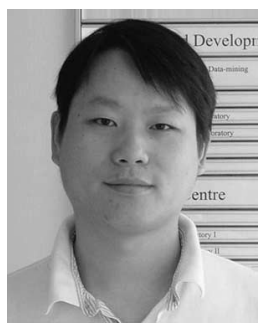

Zheng Sun (M'04) received the B.S. degree in computer science from Fudan University, Shanghai, China, in 1995, and the M.S. and Ph.D. degrees in computer science from Duke University, Durham, NC, in 1998 and 2003, respectively.

From 1999 to 2002, he was with Microsoft Corporation and Perfect Commerce as a Software Developer. In 2003, he was with the Hong Kong Baptist University, Hong Kong, where he was an Assistant Professor of computer science. He has been with Google Inc., Mountain View, CA, since 2005. His research interests include robotic motion planning, computational geometry, and data mining and its applications in e-commerce.

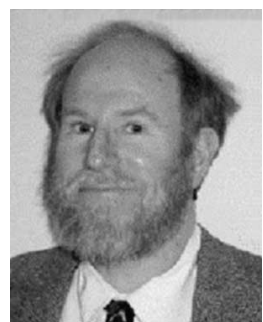

John H. Reif (S'77-M'78-SM'92-F'93) received the B.S. degree (magna cum laude) in applied math and computer science from Tufts University, Medford, MA, in 1973, and the M.S. and Ph.D. degrees in applied mathematics from Harvard University, Cambridge, MA, in 1975 and 1977, respectively.

He was an Associate Professor at Harvard University. He is currently a Hollis Edens Distinguished Professor in the Trinity College of Arts and Sciences, Duke University, Durham, NC, since 2003 and a Professor of computer science at Duke University since 1986. He is the author of over 200 papers and has edited three books on the synthesis of parallel and randomized algorithms. Although originally primarily a theoretical computer scientist, he has also made a number of contributions to practical areas of computer science including parallel architectures, data compression, robotics, and optical computing. He has also worked for many years on the development and analysis of parallel algorithms for various fundamental problems including the solution of large sparse systems, sorting, graph problems, data compression, etc. He has developed algorithms and lower bounds for a large variety of robotic motion planning problems and provided the first known computational complexity results for a robotic motion planning problem. He has also developed a wide range of efficient parallel algorithms, particularly randomized parallel algorithms. Recently, he worked on DNA computing and DNA nanostructures.

Dr. Reif is a Fellow of the Institute of Combinatorics since 1991, the Association for Computing Machinery since 1996, and the Association for the Advancement of Science since 2003. 